\title{
Some Paranormed Double Difference Sequence Spaces for Orlicz Functions and Bounded-Regular Matrices
}

\author{
S. A. Mohiuddine, ${ }^{1}$ Kuldip Raj, ${ }^{2}$ and Abdullah Alotaibi ${ }^{1}$ \\ ${ }^{1}$ Department of Mathematics, Faculty of Science, King Abdulaziz University, P.O. Box 80203, \\ Jeddah 21589, Saudi Arabia \\ ${ }^{2}$ School of Mathematics, Shri Mata Vaishno Devi University, Katra, Jammu and Kashmir 182320, India \\ Correspondence should be addressed to S. A. Mohiuddine; mohiuddine@gmail.com
}

Received 23 November 2013; Accepted 14 January 2014; Published 10 March 2014

Academic Editor: M. Mursaleen

Copyright (c) 2014 S. A. Mohiuddine et al. This is an open access article distributed under the Creative Commons Attribution License, which permits unrestricted use, distribution, and reproduction in any medium, provided the original work is properly cited.

The aim of this paper is to introduce some new double difference sequence spaces with the help of the Musielak-Orlicz function $\mathscr{F}=\left(F_{j k}\right)$ and four-dimensional bounded-regular (shortly, $R H$-regular) matrices $A=\left(a_{n m j k}\right)$. We also make an effort to study some topological properties and inclusion relations between these double difference sequence spaces.

\section{Introduction, Notations, and Preliminaries}

In [1], Hardy introduced the concept of regular convergence for double sequences. Some important work on double sequences is also found by Bromwich [2]. Later on, it was studied by various authors, for example, Móricz [3], Móricz and Rhoades [4], Başarır and Sonalcan [5], Mursaleen and Mohiuddine [6-8], and many others. Mursaleen [9] has defined and characterized the notion of almost strong regularity of four-dimensional matrices and applied these matrices to establish a core theorem (also see [10,11]). Altay and Başar [12] have recently introduced the double sequence spaces $\mathscr{B} \mathcal{S}, \mathscr{B} \mathcal{S}(t), \mathscr{C} \mathcal{S}_{p}, \mathscr{C} \mathcal{S}_{b p}, \mathscr{C} \mathcal{S}_{r}$, and $\mathscr{B} \mathscr{V}$ consisting of all double series whose sequence of partial sums are in the spaces $\mathscr{M}_{u}, \mathscr{M}_{u}(t), \mathscr{C}_{p}, \mathscr{C}_{b p}, \mathscr{C}_{r}$, and $\mathscr{L}_{u}$, respectively. Başar and Sever [13] extended the well-known space $\ell_{q}$ from single sequence to double sequences, denoted by $\mathscr{L}_{q}$, and established its interesting properties. The authors of [14] defined some convex and paranormed sequences spaces and presented some interesting characterization. Most recently, Mohiuddine and Alotaibi [15] introduced some new double sequences spaces for $\sigma$-convergence of double sequences and invariant mean and also determined some inclusion results for these spaces. For more details on these concepts, one can be referred to [16-18].
The notion of difference sequence spaces was introduced by Kizmaz [19], who studied the difference sequence spaces $l_{\infty}(\Delta), c(\Delta)$, and $c_{0}(\Delta)$. The notion was further generalized by Et and Çolak [20] by introducing the spaces $l_{\infty}\left(\Delta^{r}\right), c\left(\Delta^{r}\right)$, and $c_{0}\left(\Delta^{r}\right)$.

Let $w$ be the space of all complex or real sequences $x=$ $\left(x_{k}\right)$ and let $r$ and $s$ be two nonnegative integers. Then for $Z=$ $l_{\infty}, c, c_{0}$, we have the following sequence spaces:

$$
Z\left(\Delta_{s}^{r}\right)=\left\{x=\left(x_{k}\right) \in w:\left(\Delta_{s}^{r} x_{k}\right) \in Z\right\},
$$

where $\Delta_{s}^{r} x=\left(\Delta_{s}^{r} x_{k}\right)=\left(\Delta_{s}^{r-1} x_{k}-\Delta_{s}^{r-1} x_{k+1}\right)$ and $\Delta^{0} x_{k}=x_{k}$ for all $k \in \mathbb{N}$, which is equivalent to the following binomial representation:

$$
\Delta_{s}^{r} x_{k}=\sum_{v=0}^{r}(-1)^{v}\left(\begin{array}{l}
r \\
v
\end{array}\right) x_{k+s v} .
$$

We remark that for $s=1$ and $r=s=1$, we obtain the sequence spaces which were introduced and studied by Et and Çolak [20] and Kızmaz [19], respectively. For more details about sequence spaces see [21-27] and references therein. 
An Orlicz function $F:[0, \infty) \rightarrow[0, \infty)$ is continuous, nondecreasing, and convex such that $F(0)=0, F(x)>0$ for $x>0$ and $F(x) \rightarrow \infty$ as $x \rightarrow \infty$. If convexity of Orlicz function is replaced by $F(x+y) \leq F(x)+F(y)$, then this function is called modulus function. Lindenstrauss and Tzafriri [28] used the idea of Orlicz function to define the following sequence space:

$$
\ell_{F}=\left\{x=\left(x_{k}\right) \in w: \sum_{k=1}^{\infty} F\left(\frac{\left|x_{k}\right|}{\rho}\right)<\infty, \rho>0\right\},
$$

which is known as an Orlicz sequence space. The space $\ell_{F}$ is a Banach space with the norm

$$
\|x\|=\inf \left\{\rho>0: \sum_{k=1}^{\infty} F\left(\frac{\left|x_{k}\right|}{\rho}\right) \leq 1\right\} .
$$

Also it was shown in [28] that every Orlicz sequence space $\ell_{F}$ contains a subspace isomorphic to $\ell_{p}(p \geq 1)$. An Orlicz function $F$ can always be represented in the following integral form:

$$
F(x)=\int_{0}^{x} \eta(t) d t
$$

where $\eta$ is known as the kernel of $F$, is a right differentiable for $t \geq 0, \eta(0)=0, \eta(t)>0, \eta$ is nondecreasing, and $\eta(t) \rightarrow$ $\infty$ as $t \rightarrow \infty$.

A sequence $\mathscr{F}=\left(F_{k}\right)$ of Orlicz functions is said to be a Musielak-Orlicz function (see [29,30]). A sequence $\mathcal{N}=\left(N_{k}\right)$ is defined by

$$
N_{k}(v)=\sup \left\{|v| u-F_{k}(u): u \geq 0\right\}, \quad k=1,2, \ldots,
$$

which is called the complementary function of a MusielakOrlicz function $\mathscr{F}$. For a given Musielak-Orlicz function $\mathscr{F}$, the Musielak-Orlicz sequence space $t_{\mathscr{F}}$ and its subspace $h_{\mathscr{F}}$ are defined as follows:

$$
\begin{gathered}
t_{\mathscr{F}}=\left\{x \in w: I_{\mathscr{F}}(c x)<\infty \text { for some } c>0\right\}, \\
h_{\mathscr{F}}=\left\{x \in w: I_{\mathscr{F}}(c x)<\infty \forall c>0\right\},
\end{gathered}
$$

where $I_{\mathscr{F}}$ is a convex modular defined by

$$
I_{\mathscr{F}}(x)=\sum_{k=1}^{\infty} F_{k}\left(x_{k}\right), \quad x=\left(x_{k}\right) \in t_{\mathscr{F}} .
$$

We consider $t_{\mathscr{F}}$ equipped with the Luxemburg norm

$$
\|x\|=\inf \left\{k>0: I_{\mathscr{F}}\left(\frac{x}{k}\right) \leq 1\right\}
$$

or equipped with the Orlicz norm

$$
\|x\|^{0}=\inf \left\{\frac{1}{k}\left(1+I_{\mathscr{F}}(k x)\right): k>0\right\} .
$$

A Musielak-Orlicz function $\mathscr{F}=\left(F_{k}\right)$ is said to satisfy $\Delta_{2}$-condition if there exist constants $a, K>0$ and a sequence $c=\left(c_{k}\right)_{k=1}^{\infty} \in l_{+}^{1}$ (the positive cone of $l^{1}$ ) such that the inequality

$$
F_{k}(2 u) \leq K F_{k}(u)+c_{k}
$$

holds for all $k \in \mathbb{N}$ and $u \in \mathbb{R}^{+}$, whenever $F_{k}(u) \leq a$.

A double sequence $x=\left(x_{j k}\right)$ is said to be bounded if $\|x\|_{(\infty, 2)}=\sup _{j, k}\left|x_{j k}\right|<\infty$. We denote by $l_{\infty}^{2}$ the space of all bounded double sequences.

By the convergence of double sequence $x=\left(x_{j k}\right)$ we mean the convergence in the Pringsheim sense; that is, a double sequence $x=\left(x_{j k}\right)$ is said to converge to the limit $L$ in Pringsheim sense (denoted by, $P-\lim x=L$ ) provided that given $\epsilon>0$ there exists $n \in \mathbb{N}$ such that $\left|x_{j k}-L\right|<\epsilon$ whenever $j, k>n$ (see [31]). We will write more briefly as $P$-convergent. If, in addition, $x \in l_{\infty}^{2}$, then $x$ is said to be boundedly P-convergent to $L$. We will denote the space of all bounded convergent double sequences (or boundedly $P$ convergent) by $c_{\infty}^{2}$.

Let $S \subseteq \mathbb{N} \times \mathbb{N}$ and let $\epsilon>0$ be given. By $\chi_{S(x ; \epsilon)}$, we denote the characteristic function of the set $S(x ; \epsilon)=\{(j, k) \in \mathbb{N} \times \mathbb{N}$ : $\left.\left|x_{j k}\right| \geq \epsilon\right\}$.

Let $A=\left(a_{n m j k}\right)$ be a four-dimensional infinite matrix of scalers. For all $m, n \in \mathbb{N}_{0}$, where $\mathbb{N}_{0}:=\mathbb{N} \cup\{0\}$, the sum

$$
y_{n m}=\sum_{j, k=0,0}^{\infty, \infty} a_{n m j k} x_{j k}
$$

is called the $A$-means of the double sequence $\left(x_{j k}\right)$. A double sequence $\left(x_{j k}\right)$ is said to be $A$-summable to the limit $L$ if the $A$-means exist for all $m, n$ in the sense of Pringsheim's convergence:

$$
P-\lim _{p, q \rightarrow \infty} \sum_{j, k=0,0}^{p, q} a_{n m j k} x_{j k}=y_{n m}, \quad P-\lim _{n, m \rightarrow \infty} y_{n m}=L .
$$

A four-dimensional matrix $A$ is said to be boundedregular (or $R H$-regular) if every bounded $P$-convergent sequence is $A$-summable to the same limit and the $A$-means are also bounded.

The following is a four-dimensional analogue of the wellknown Silverman-Toeplitz theorem [32].

Theorem 1 (Robison [33] and Hamilton [34]). The fourdimensional matrix $A$ is $R H$-regular if and only if

$\left(\mathrm{RH}_{1}\right) P$ - $\lim _{n, m} a_{n m j k}=0$ for each $j$ and $k$,

$\left(\mathrm{RH}_{2}\right) P-\lim _{n, m} \sum_{j, k=0,0}^{\infty, \infty}\left|a_{n m j k}\right|=1$,

$\left(\mathrm{RH}_{3}\right) P-\lim _{n, m} \sum_{j=0}^{\infty}\left|a_{n m j k}\right|=0$ for each $k$,

$\left(\mathrm{RH}_{4}\right) P-\lim _{n, m} \sum_{k=0}^{\infty}\left|a_{n m j k}\right|=0$ for each $j$,

$\left(\mathrm{RH}_{5}\right) \sum_{j, k=0,0}^{\infty, \infty}\left|a_{n m j k}\right|<\infty$ for all $n, m \in \mathbb{N}_{0}$. 


\section{The Double Difference Sequence Spaces}

In this section, we define some new paranormed double difference sequence spaces with the help of Musielak-Orlicz functions and four-dimensional bounded-regular matrices. Before proceeding further, first we recall the notion of paranormed space as follows.

A linear topological space $X$ over the real field $\mathbb{R}$ (the set of real numbers) is said to be a paranormed space if there is a subadditive function $g: X \rightarrow \mathbb{R}$ such that $g(\theta)=0, g(x)=g(-x)$, and scalar multiplication is continuous; that is, $\left|\alpha_{n}-\alpha\right| \rightarrow 0$ and $g\left(x_{n}-x\right) \rightarrow$ 0 imply $g\left(\alpha_{n} x_{n}-\alpha x \mid \rightarrow 0\right.$ for all $\alpha$ 's in $\mathbb{R}$ and all $x$ 's in $X$, where $\theta$ is the zero vector in the linear space $X$.

The linear spaces $l_{\infty}(p), c(p)$, and $c_{0}(p)$ were defined by Maddox [35] (also, see Simons [36]).

Let $\mathscr{F}=\left(F_{j k}\right)$ be a Musielak-Orlicz function; that is, $\mathscr{F}$ is a sequence of Orlicz functions and let $A=\left(a_{n m j k}\right)$ be a nonnegative four-dimensional bounded-regular matrix. Then, we define the following:

$$
\begin{aligned}
& W_{0}^{2}\left(A, \mathscr{F}, u, \Delta_{s}^{r}, p\right) \\
& =\left\{x=\left(x_{j k}\right):\right. \\
& \left.\quad P-\lim _{n, m} \sum_{j, k=0,0}^{\infty, \infty} a_{n m j k}\left[F_{j k}\left(u_{j k}\left|\Delta_{s}^{r} x_{j k}\right|\right)^{p_{j k}}\right]=0\right\},
\end{aligned}
$$$$
W^{2}\left(A, \mathscr{F}, u, \Delta_{s}^{r}, p\right)
$$

$$
\begin{aligned}
& =\left\{x=\left(x_{j k}\right):\right. \\
& P-\lim _{n, m} \sum_{j, k=0,0}^{\infty, \infty} a_{n m j k}\left[F_{j k}\left(u_{j k}\left|\Delta_{s}^{r} x_{j k}-L\right|\right)^{p_{j k}}\right] \\
& =0 \text { for some } L \in \mathbb{C}\},
\end{aligned}
$$

where $p=\left(p_{j k}\right)$ is a double sequence of real numbers such that $p_{j k}>0$ for $j, k, \sup _{j, k} p_{j k}=H<\infty$, and $u=\left(u_{j k}\right)$ is a double sequence of strictly positive real numbers.
Remark 2. If we take $\mathscr{F}(x)=x$ in $W_{0}^{2}\left(A, \mathscr{F}, u, \Delta_{s}^{r}, p\right)$ and $W^{2}\left(A, \mathscr{F}, u, \Delta_{s}^{r}, p\right)$, then we have the following spaces:

$$
\begin{aligned}
& W_{0}^{2}\left(A, u, \Delta_{s}^{r}, p\right) \\
& =\left\{x=\left(x_{j k}\right):\right. \\
& \left.P-\lim _{n, m} \sum_{j, k=0,0}^{\infty, \infty} a_{n m j k}\left[\left(u_{j k}\left|\Delta_{s}^{r} x_{j k}\right|\right)^{p_{j k}}\right]=0\right\}, \\
& W^{2}\left(A, u, \Delta_{s}^{r}, p\right) \\
& =\left\{x=\left(x_{j k}\right):\right. \\
& P-\lim \sum_{n, m}^{\infty, \infty} a_{j, k=0,0}\left[\left(u_{j k}\left|\Delta_{s}^{r} x_{j k}-L\right|\right)^{p_{j k}}\right] \\
& =0 \text { for some } L \in \mathbb{C}\} \text {. }
\end{aligned}
$$

Remark 3. Let $p=\left(p_{j k}\right)=1$ for all $j, k$. Then $W_{0}^{2}(A, \mathscr{F}, u$, $\left.\Delta_{s}^{r}, p\right)$ and $W^{2}\left(A, \mathscr{F}, u, \Delta_{s}^{r}, p\right)$ are reduced to

$$
\begin{aligned}
& W_{0}^{2}\left(A, \mathscr{F}, u, \Delta_{s}^{r}\right) \\
& =\left\{x=\left(x_{j k}\right):\right. \\
& \left.P-\lim _{n, m} \sum_{j, k=0,0}^{\infty, \infty} a_{n m j k}\left[F_{j k}\left(u_{j k}\left|\Delta_{s}^{r} x_{j k}\right|\right)\right]=0\right\}, \\
& W^{2}\left(A, \mathscr{F}, u, \Delta_{s}^{r}\right) \\
& =\left\{x=\left(x_{j k}\right):\right. \\
& P-\lim _{n, m} \sum_{j, k=0,0}^{\infty, \infty} a_{n m j k}\left[F_{j k}\left(u_{j k}\left|\Delta_{s}^{r} x_{j k}-L\right|\right)\right] \\
& =0 \text { for some } L \in \mathbb{C}\} \text {, }
\end{aligned}
$$

respectively. 
Remark 4. Let $u=\left(u_{j k}\right)=1$ for all $j, k$. Then, the spaces $W_{0}^{2}\left(A, \mathscr{F}, u, \Delta_{s}^{r}, p\right)$ and $W^{2}\left(A, \mathscr{F}, u, \Delta_{s}^{r}, p\right)$ are reduced to

$$
\begin{aligned}
& W_{0}^{2}\left(A, \mathscr{F}, \Delta_{s}^{r}, p\right) \\
& =\left\{x=\left(x_{j k}\right):\right. \\
& \left.\quad P-\lim _{n, m} \sum_{j, k=0,0}^{\infty, \infty} a_{n m j k}\left[F_{j k}\left(\left|\Delta_{s}^{r} x_{j k}\right|\right)^{p_{j k}}\right]=0\right\}, \\
& W^{2}\left(A, \mathscr{F}, \Delta_{s}^{r}, p\right) \\
& =\left\{\begin{array}{c}
x=\left(x_{j k}\right): \\
P-\lim _{n, m} \sum_{j, k=0,0}^{\infty, \infty} a_{n m j k}\left[F_{j k}\left(\left|\Delta_{s}^{r} x_{j k}-L\right|\right)^{p_{j k}}\right] \\
=0 \text { for some } L \in \mathbb{C}\},
\end{array}\right.
\end{aligned}
$$

respectively.

Remark 5. If we take $A=(C, 1,1)$ in $W_{0}^{2}\left(A, \mathscr{F}, u, \Delta_{s}^{r}, p\right)$ and $W^{2}\left(A, \mathscr{F}, u, \Delta_{s}^{r}, p\right)$, then we have the following spaces:

$$
\begin{aligned}
& W_{0}^{2}\left(\mathscr{F}, u, \Delta_{s}^{r}, p\right) \\
& =\left\{x=\left(x_{j k}\right):\right. \\
& \left.P-\lim _{n, m} \sum_{j, k=0,0}^{m-1, n-1}\left[F_{j k}\left(u_{j k}\left|\Delta_{s}^{r} x_{j k}\right|\right)^{p_{j k}}\right]=0\right\}, \\
& W^{2}\left(\mathscr{F}, u, \Delta_{s}^{r}, p\right) \\
& =\left\{x=\left(x_{j k}\right):\right. \\
& P-\lim _{n, m} \sum_{j, k=0,0}^{m-1, n-1}\left[F_{j k}\left(u_{j k}\left|\Delta_{s}^{r} x_{j k}-L\right|\right)^{p_{j k}}\right] \\
& =0 \text { for some } L \in \mathbb{C}\} \text {. }
\end{aligned}
$$

Remark 6. If we take $A=(C, 1,1)$ and $\mathscr{F}(x)=x$ in $W_{0}^{2}\left(A, \mathscr{F}, u, \Delta_{s}^{r}, p\right)$ and $W^{2}\left(A, \mathscr{F}, u, \Delta_{s}^{r}, p\right)$, then we have the following spaces:

$$
\begin{aligned}
& W_{0}^{2}\left(u, \Delta_{s}^{r}, p\right) \\
& =\left\{x=\left(x_{j k}\right):\right. \\
& \left.P-\lim _{n, m} \sum_{j, k=0,0}^{m-1, n-1}\left[\left(u_{j k}\left|\Delta_{s}^{r} x_{j k}\right|\right)^{p_{j k}}\right]=0\right\}, \\
& W^{2}\left(u, \Delta_{s}^{r}, p\right) \\
& =\left\{x=\left(x_{j k}\right):\right. \\
& P-\lim _{n, m} \sum_{j, k=0,0}^{m-1, n-1}\left[\left(u_{j k}\left|\Delta_{s}^{r} x_{j k}-L\right|\right)^{p_{j k}}\right] \\
& =0 \text { for some } L \in \mathbb{C}\} \text {. }
\end{aligned}
$$

Remark 7. Let $p_{j k}=u_{j k}=1$ for all $j, k$. If, in addition, $\mathscr{F}(x)=$ $F(x)$ and $r=0$, then the spaces $W_{0}^{2}\left(A, \mathscr{F}, u, \Delta_{s}^{r}, p\right)$ and $W^{2}\left(A, \mathscr{F}, u, \Delta_{s}^{r}, p\right)$ are reduced to $W_{0}^{2}(A, F)$ and $W^{2}(A, F)$ which were introduced and studied by Yurdakadim and Tas [37] as below:

$$
\begin{gathered}
W_{0}^{2}(A, F)=\left\{x=\left(x_{j k}\right): P-\lim _{n, m} \sum_{j, k} a_{n m j k} F\left(\left|x_{j k}\right|\right)=0\right\}, \\
W^{2}(A, F)=\left\{x=\left(x_{j k}\right): P-\lim _{n, m} \sum_{j, k} a_{n m j k} F\left(\left|x_{j k}-L\right|\right)\right. \\
=0 \text { for some } L \in \mathbb{C}\} .
\end{gathered}
$$

Throughout the paper, we will use the following inequality: let $\left(a_{j k}\right)$ and $\left(b_{j k}\right)$ be two double sequences. Then

$$
\left|a_{j k}+b_{j k}\right|^{p_{j k}} \leq K\left(\left|a_{j k}\right|^{p_{j k}}+\left|b_{j k}\right|^{p_{j k}}\right),
$$

where $K=\max \left(1,2^{H-1}\right)$ and $\sup _{j, k} p_{j k}=H$ (see [15]). We will also assume throughout this paper that the symbol $\mathscr{F}$ will denote the sublinear Musielak-Orlicz function. 


\section{Main Results}

Theorem 8. Let $\mathscr{F}=\left(F_{j k}\right)$ be a sublinear Musielak-Orlicz function, $A=\left(a_{n m j k}\right)$ a nonnegative four-dimensional $R H$ regular matrix, $p=\left(p_{j k}\right)$ a bounded sequence of positive real numbers, and $u=\left(u_{j k}\right)$ a sequence of strictly positive real numbers. Then $W_{0}^{2}\left(A, \mathscr{F}, u, \Delta_{s}^{r}, p\right)$ and $W^{2}\left(A, \mathscr{F}, u, \Delta_{s}^{r}, p\right)$ are linear spaces over the complex field $\mathbb{C}$.

Proof. Let $x=\left(x_{j k}\right), y=\left(y_{j k}\right) \in W_{0}^{2}\left(A, \mathscr{F}, u, \Delta_{s}^{r}, p\right)$ and $\alpha, \beta \in \mathbb{C}$. Then there exist integers $M_{\alpha}$ and $N_{\beta}$ such that $|\alpha| \leq$ $M_{\alpha}$ and $|\beta| \leq N_{\beta}$.

Since $\mathscr{F}=\left(F_{j k}\right)$ is a nondecreasing function, so by inequality (21), we have

$$
\begin{aligned}
\sum_{j, k=0,0}^{\infty, \infty} a_{n m j k}\left[F_{j k}\left(u_{j k}\left|\Delta_{s}^{r}\left(\alpha x_{j k}+\beta y_{j k}\right)\right|\right)^{p_{j k}}\right] \\
\leq \sum_{j, k=0,0}^{\infty, \infty} a_{n m j k}\left[F_{j k}\left(u_{j k}\left|\alpha \Delta_{s}^{r} x_{j k}+\beta \Delta_{s}^{r} y_{j k}\right|\right)^{p_{j k}}\right] \\
\leq K \sum_{j, k=0,0}^{\infty, \infty} a_{n m j k}\left[F_{j k} M_{\alpha}\left(u_{j k}\left|\Delta_{s}^{r} x_{j k}\right|\right)^{p_{j k}}\right] \\
+K \sum_{j, k=0,0}^{\infty, \infty} a_{n m j k}\left[F_{j k} N_{\beta}\left(u_{j k}\left|\Delta_{s}^{r} y_{j k}\right|\right)^{p_{j k}}\right] \\
\leq K M_{\alpha}^{H} \sum_{j, k=0,0}^{\infty, \infty} a_{n m j k}\left[F_{j k}\left(u_{j k}\left|\Delta_{s}^{r} x_{j k}\right|\right)^{p_{j k}}\right] \\
+K N_{\beta}^{H} \sum_{j, k=0,0}^{\infty, \infty} a_{n m j k}\left[F_{j k}\left(u_{j k}\left|\Delta_{s}^{r} y_{j k}\right|\right)^{p_{j k}}\right] \longrightarrow 0 .
\end{aligned}
$$

Thus $\alpha x+\beta y \in W_{0}^{2}\left(A, \mathscr{F}, u, \Delta_{s}^{r}, p\right)$. This proves that $W_{0}^{2}\left(A, \mathscr{F}, u, \Delta_{s}^{r}, p\right)$ is a linear space. Similarly we can prove that $W^{2}\left(A, \mathscr{F}, u, \Delta_{s}^{r}, p\right)$ is also a linear space.

Theorem 9. Let $\mathscr{F}=\left(F_{j k}\right)$ be a sublinear Musielak-Orlicz function, $A=\left(a_{n m j k}\right)$ a nonnegative four-dimensional $\mathrm{RH}$ regular matrix, $p=\left(p_{j k}\right)$ a bounded sequence of positive real numbers, and $u=\left(u_{j k}\right)$ a sequence of strictly positive real numbers. Then $W_{0}^{2}\left(A, \mathscr{F}, u, \Delta_{s}^{r}, p\right)$ and $W^{2}\left(A, \mathscr{F}, u, \Delta_{s}^{r}, p\right)$ are paranormed spaces with the paranorm

$$
g(x)=\sup _{n, m} \sum_{j, k=0,0}^{\infty, \infty}\left\{a_{n m j k}\left[F_{j k}\left(u_{j k}\left|\Delta_{s}^{r} x_{j k}\right|\right)^{p_{j k}}\right]\right\}^{1 / M},
$$

where $0<p_{j k} \leq \sup p_{j k}=H<\infty$ and $M=\max (1, H)$.

Proof. We will prove the result for $W_{0}^{2}\left(A, \mathscr{F}, u, \Delta_{s}^{r}, p\right)$. Let $x=\left(x_{j k}\right) \in W_{0}^{2}\left(A, \mathscr{F}, u, \Delta_{s}^{r}, p\right)$. Then for each $x=\left(x_{j k}\right) \in$ $W_{0}^{2}\left(A, \mathscr{F}, u, \Delta_{s}^{r}, p\right), g(x)$ exists. Also it is clear that $g(0)=$ $0, g(-x)=g(x)$, and $g(x+y) \leq g(x)+g(y)$.
We now show that the scalar multiplication is continuous. First observe the following:

$$
\begin{aligned}
g(\lambda x) & =\sup _{n m} \sum_{j, k=0,0}^{\infty, \infty} a_{n m j k}\left[F_{j k}\left(u_{j k}\left|\lambda \Delta_{s}^{r} x_{j k}\right|\right)^{p_{j k}}\right] \\
& \leq(1+[|\lambda|]) g(x),
\end{aligned}
$$

where $[|\lambda|]$ denotes the integer part of $|\lambda|$. It is also clear that if $x \rightarrow 0$ and $\lambda \rightarrow 0$ implies $g(\lambda x) \rightarrow 0$. For fixed $\lambda$, if $x \rightarrow 0$, then $g(\lambda x) \rightarrow 0$. We need to show that for fixed $x, \lambda \rightarrow 0$ implies $g(\lambda x) \rightarrow 0$. Let $x \in W^{2}\left(A, \mathscr{F}, u, \Delta_{s}^{r}, p\right)$. Thus

$$
P-\lim _{n, m} \sum_{j, k=0,0}^{\infty, \infty} a_{n m j k}\left[F_{j k}\left(u_{j k}\left|\Delta_{s}^{r} x_{j k}-L\right|\right)^{p_{j k}}\right]=0 .
$$

Then, for $\epsilon>0$ there exists $N \in \mathbb{N}$ such that

$$
\sum_{j, k=0,0}^{\infty, \infty} a_{n m j k}\left[F_{j k}\left(u_{j k}\left|\Delta_{s}^{r} x_{j k}-L\right|\right)^{p_{j k}}\right]<\frac{\epsilon}{4}
$$

for $m, n>N$. Also, for each $m, n$ with $1 \leq m, n \leq N$, since

$$
\sum_{j, k=0,0}^{\infty, \infty} a_{n m j k}\left[F_{j k}\left(u_{j k}\left|\Delta_{s}^{r} x_{j k}-L\right|\right)^{p_{j k}}\right]<\infty
$$

there exists an integer $M_{m, n}$ such that

$$
\sum_{j, k>M_{m, n}} a_{n m j k}\left[F_{j k}\left(u_{j k}\left|\Delta_{s}^{r} x_{j k}-L\right|\right)^{p_{j k}}\right]<\frac{\epsilon}{4} .
$$

Let $M=\max _{1 \leq(m, n) \leq N}\left\{M_{m, n}\right\}$. We have for each $m, n$ with $1 \leq$ $m, n \leq N$

$$
\sum_{j, k>M} a_{n m j k}\left[F_{j k}\left(u_{j k}\left|\Delta_{s}^{r} x_{j k}-L\right|\right)^{p_{j k}}\right]<\frac{\epsilon}{4} .
$$

Also from (26), for $m, n>N$, we have

$$
\sum_{j, k>M} a_{n m j k}\left[F_{j k}\left(u_{j k}\left|\Delta_{s}^{r} x_{j k}-L\right|\right)^{p_{j k}}\right]<\frac{\epsilon}{4} .
$$

Thus $M$ is an integer independent of $m, n$ such that

$$
\sum_{j, k>M} a_{n m j k}\left[F_{j k}\left(u_{j k}\left|\Delta_{s}^{r} x_{j k}-L\right|\right)^{p_{j k}}\right]<\frac{\epsilon}{4} .
$$


Since $|\lambda|^{p_{j k}} \leq \max \left(1,|\lambda|^{H}\right)$, therefore

$$
\begin{aligned}
\sum_{j, k=0,0}^{\infty, \infty} a_{n m j k}\left[F_{j k}\left(u_{j k}\left|\lambda \Delta_{s}^{r} x_{j k}\right|\right)^{p_{j k}}\right] \\
=\sum_{j, k=0,0}^{\infty, \infty} a_{n m j k}\left[F_{j k}\left(u_{j k}\left|\lambda \Delta_{s}^{r} x_{j k}-\lambda L+\lambda L\right|\right)^{p_{j k}}\right] \\
\leq \sum_{j, k=0,0}^{\infty, \infty} a_{n m j k}\left[F_{j k}\left(u_{j k}\left|\lambda \Delta_{s}^{r} x_{j k}-\lambda L\right|\right)^{p_{j k}}\right] \\
+\sum_{j, k=0,0}^{\infty, \infty} a_{n m j k}\left[F_{j k}\left(u_{j k}|\lambda L|\right)^{p_{j k}}\right] \\
\leq \sum_{j, k>M} a_{n m j k}\left[F_{j k}\left(u_{j k}\left|\lambda \Delta_{s}^{r} x_{j k}-\lambda L\right|\right)^{p_{j k}}\right] \\
\quad+\sum_{j, k \leq M} a_{n m j k}\left[F_{j k}\left(u_{j k}\left|\lambda \Delta_{s}^{r} x_{j k}-\lambda L\right|\right)^{p_{j k}}\right] \\
\quad+\sum_{j \geq M, k<M} a_{n m j k}\left[F_{j k}\left(u_{j k}\left|\lambda \Delta_{s}^{r} x_{j k}-\lambda L\right|\right)^{p_{j k}}\right] \\
+\sum_{j, k=0,0}^{\infty, \infty} a_{n m j k}\left[F_{j k}\left(u_{j k}|\lambda L|\right)^{p_{j k}}\right] . \\
+\sum_{n m j k}\left[F_{j k}\left(u_{j k}\left|\lambda \Delta_{s}^{r} x_{j k}-\lambda L\right|\right)^{p_{j k}}\right]
\end{aligned}
$$

For each $m, n$ and by the continuity of $F$ as $\lambda \rightarrow 0$, we have the following:

$$
\begin{aligned}
\sum_{j, k \leq M} a_{n m j k}\left[F_{j k}\left(u_{j k}\left|\lambda \Delta_{s}^{r} x_{j k}-\lambda L\right|\right)^{p_{j k}}\right] & \\
+\sum_{j, k=0,0}^{\infty, \infty} a_{n m j k}\left[F_{j k}\left(u_{j k}|\lambda L|\right)^{p_{j k}}\right] & \longrightarrow 0
\end{aligned}
$$

in Pringsheim's sense. Now choose $\delta<1$ such that $|\lambda|<\delta$ implies

$$
\begin{aligned}
\sum_{j, k \leq M} a_{n m j k}\left[F_{j k}\left(u_{j k}\left|\lambda \Delta_{s}^{r} x_{j k}-\lambda L\right|\right)^{p_{j k}}\right] \\
+\sum_{j, k=0,0}^{\infty, \infty} a_{n m j k}\left[F_{j k}\left(u_{j k}|\lambda L|\right)^{p_{j k}}\right]<\frac{\epsilon}{4} .
\end{aligned}
$$

In the same manner, we have

$$
\begin{gathered}
\sum_{j \geq M, k<M} a_{n m j k}\left[F_{j k}\left(u_{j k}\left|\lambda \Delta_{s}^{r} x_{j k}-\lambda L\right|\right)^{p_{j k}}\right]<\frac{\epsilon}{4}, \\
\sum_{j<M, k \geq M} a_{n m j k}\left[F_{j k}\left(u_{j k}\left|\lambda \Delta_{s}^{r} x_{j k}-\lambda L\right|\right)^{p_{j k}}\right]<\frac{\epsilon}{4} .
\end{gathered}
$$

It follows from (31), (34), (35), and (36) that

$$
\sum_{j, k=0,0}^{\infty, \infty} a_{n m j k}\left[F_{j k}\left(u_{j k}\left|\lambda \Delta_{s}^{r} x_{j k}\right|\right)^{p_{j k}}\right]<\epsilon \quad \forall m, n .
$$

Thus $g(\lambda x) \rightarrow 0$ as $\lambda \rightarrow 0$. Therefore $W_{0}^{2}\left(A, \mathscr{F}, u, \Delta_{s}^{r}, p\right)$ is a paranormed space. Similarly, we can prove that $W^{2}\left(A, \mathscr{F}, u, \Delta_{s}^{r}, p\right)$ is a paranormed space. This completes the proof.

Theorem 10. Let $\mathscr{F}=\left(F_{j k}\right)$ be a sublinear Musielak-Orlicz function, $A=\left(a_{n m j k}\right)$ a nonnegative four-dimensional $\mathrm{RH}$ regular matrix, $p=\left(p_{j k}\right)$ a bounded sequence of positive real numbers, and $u=\left(u_{j k}\right)$ a sequence of strictly positive real numbers. Then $W_{0}^{2}\left(A, \mathscr{F}, u, \Delta_{s}^{r}, p\right)$ and $W^{2}\left(A, \mathscr{F}, u, \Delta_{s}^{r}, p\right)$ are complete topological linear spaces.

Proof. Let $\left(x_{j k}^{q}\right)$ be a Cauchy sequence in $W_{0}^{2}\left(A, \mathscr{F}, u, \Delta_{s}^{r}, p\right)$; that is, $g\left(x^{q}-x^{t}\right) \rightarrow 0$ as $q, t \rightarrow \infty$. Then, we have

$$
\sum_{j, k=0,0}^{\infty, \infty} a_{n m j k}\left[F_{j k}\left(u_{j k}\left|\Delta_{s}^{r} x_{j k}^{q}-\Delta_{s}^{r} x_{j k}^{t}\right|\right)^{p_{j k}}\right] \longrightarrow 0
$$

Thus for each fixed $j$ and $k$ as $q, t \rightarrow \infty$, since $A=\left(a_{n m j k}\right)$ is nonnegative, we are granted that

$$
F_{j k}\left(u_{j k}\left|\Delta_{s}^{r} x_{j k}^{q}-\Delta_{s}^{r} x_{j k}^{t}\right|\right) \longrightarrow 0
$$

and by continuity of $\mathscr{F}=\left(F_{j k}\right),\left(x_{j k}^{q}\right)$ is a Cauchy sequence in $\mathbb{C}$ for each fixed $j$ and $k$.

Since $\mathbb{C}$ is complete as $t \rightarrow \infty$, we have $x_{j k}^{q} \rightarrow x_{j k}$ for each $(j, k)$. Now from (36), we have that, for $\epsilon>0$, there exists a natural number $N$ such that

$$
\sum_{j, k=0,0 q, t>N}^{\infty, \infty} a_{n m j k}\left[F_{j k}\left(u_{j k}\left|\Delta_{s}^{r} x_{j k}^{q}-\Delta_{s}^{r} x_{j k}^{t}\right|\right)^{p_{j k}}\right]<\epsilon \quad \forall m, n .
$$

Since for any fixed natural number $M$, from (38) we have

$$
\sum_{j, k \leq M q, t>N}^{\infty, \infty} a_{n m j k}\left[F_{j k}\left(u_{j k}\left|\Delta_{s}^{r} x_{j k}^{q}-\Delta_{s}^{r} x_{j k}^{t}\right|\right)^{p_{j k}}\right]<\epsilon \quad \forall m, n .
$$

By letting $t \rightarrow \infty$ in the above expression we obtain

$$
\sum_{j, k \leq M}^{\infty, \infty} a_{n m j k}\left[F_{j k}\left(u_{j k}\left|\Delta_{s}^{r} x_{j k}^{q}-\Delta_{s}^{r} x_{j k}\right|\right)^{p_{j k}}\right]<\epsilon .
$$

Since $M$ is arbitrary, by letting $M \rightarrow \infty$ we obtain

$$
\sum_{j, k=0,0}^{\infty, \infty} a_{n m j k}\left[F_{j k}\left(u_{j k}\left|\Delta_{s}^{r} x_{j k}^{q}-\Delta_{s}^{r} x_{j k}\right|\right)^{p_{j k}}\right]<\epsilon \quad \forall m, n .
$$

Thus $g\left(x^{q}-x\right) \rightarrow 0$ as $q \rightarrow \infty$. This proves that $W_{0}^{2}\left(A, \mathscr{F}, u, \Delta_{s}^{r}, p\right)$ is a complete topological linear space. 
Now we will show that $W^{2}\left(A, \mathscr{F}, u, \Delta_{s}^{r}, p\right)$ is a complete topological linear space. For this, since $\left(x^{q}\right)$ is also a sequence in $W^{2}\left(A, \mathscr{F}, u, \Delta_{s}^{r}, p\right)$ by definition of $W^{2}\left(A, \mathscr{F}, u, \Delta_{s}^{r}, p\right)$, for each $q$, there exists $L^{q}$ with

$$
\sum_{j, k=0,0}^{\infty, \infty} a_{n m j k}\left[F_{j k}\left(u_{j k}\left|\Delta_{s}^{r} x_{j k}^{q}-\Delta_{s}^{r} L^{q}\right|\right)^{p_{j k}}\right] \longrightarrow 0
$$

$$
\text { as } m, n \longrightarrow \infty \text {; }
$$

whence from the fact that $\sup _{n m} \sum_{j, k=0,0}^{\infty, \infty} a_{n m j k}<\infty$ and from the definition of Musielak-Orlicz function, we have $F_{j k}\left|\Delta_{s}^{r} L^{q}-\Delta_{s}^{r} L\right| \rightarrow 0$ as $q \rightarrow \infty$ and so $L^{q}$ converges to $L$. Thus

$$
\begin{aligned}
\sum_{j, k=0,0}^{\infty, \infty} a_{n m j k}\left[F_{j k}\left(u_{j k}\left|\Delta_{s}^{r} x_{j k}-L\right|\right)^{p_{j k}}\right] & \longrightarrow 0 \\
\text { as } m, n & \longrightarrow \infty .
\end{aligned}
$$

Hence $x \in W^{2}\left(A, \mathscr{F}, u, \Delta_{s}^{r}, p\right)$ and this completes the proof.

Theorem 11. Let $\mathscr{F}=\left(F_{j k}\right)$ be a sublinear MusielakOrlicz function which satisfies the $\Delta_{2}$-condition. Then $W^{2}\left(A, u, \Delta_{s}^{r}, p\right) \subseteq W^{2}\left(A, \mathscr{F}, u, \Delta_{s}^{r}, p\right)$.

Proof. Let $x=\left(x_{k}\right) \in W^{2}\left(A, u, \Delta_{s}^{r}, p\right)$; that is,

$$
\lim _{n, m} \sum_{j, k} a_{n m j k}\left[\left(u_{j k}\left|\Delta_{s}^{r} x_{j k}-L\right|\right)^{p_{j k}}\right]=0 .
$$

Let $\epsilon>0$ and choose $\delta$ with $0<\delta<1$ such that $F_{j k}(t)<\epsilon$ for $0 \leq t \leq \delta$. Write $y_{j k}=\left(u_{j k}\left|\Delta_{s}^{r} x_{j k}-L\right|\right)$ and consider

$$
\begin{aligned}
\sum_{j, k} a_{n m j k}\left[F_{j k}\left(y_{j k}\right)^{\left.p_{j k}\right]=}\right. & \sum_{j, k:\left|y_{j k}\right| \leq \delta} a_{n m j k}\left[F_{j k}\left(y_{j k}\right)^{p_{j k}}\right] \\
& +\sum_{j, k:\left|y_{j k}\right|>\delta} a_{n m j k}\left[F_{j k}\left(y_{j k}\right)^{p_{j k}}\right] \\
= & \epsilon \sum_{j, k:\left|y_{j k}\right| \leq \delta} a_{n m j k} \\
& +\sum_{j, k:\left|y_{j k}\right|>\delta} a_{n m j k}\left[F_{j k}\left(y_{j k}\right)^{p_{j k}}\right] .
\end{aligned}
$$

For $y_{j k}>\delta$, we use the fact that $y_{j k}<y_{j k} / \delta<1+y_{j k} / \delta$. Hence

$$
F_{j k}\left(y_{j k}\right)<F_{j k}\left(1+\frac{y_{j k}}{\delta}\right)<\frac{F_{j k}(2)}{2}+\frac{1}{2} F_{j k}\left(2 \frac{y_{j k}}{\delta}\right) .
$$

Since $\mathscr{F}$ satisfies the $\Delta_{2}$-condition, we have

$$
F_{j k}\left(y_{j k}\right)<K \frac{y_{j k}}{2 \delta} F_{j k}(2)+K \frac{y_{j k}}{2 \delta} F_{j k}(2)=K \frac{y_{j k}}{\delta} F_{j k}(2),
$$

and hence

$$
\begin{aligned}
& \sum_{j, k:\left|y_{j k}\right|>\delta} a_{n m j k}\left[F_{j k}\left(y_{j k}\right)^{p_{j k}}\right] \\
& \leq K \frac{F_{j k}}{\delta}(2) \sum_{j, k} a_{n m j k}\left[\left(u_{j k}\left|\Delta_{s}^{r} x_{j k}-L\right|\right)^{p_{j k}}\right] .
\end{aligned}
$$

Since $A$ is $R H$-regular and $x \in W^{2}\left(A, u, \Delta_{s}^{r}, p\right)$, we get $x \in$ $W^{2}\left(A, \mathscr{F}, u, \Delta_{s}^{r}, p\right)$.

Theorem 12. Let $\mathscr{F}=\left(F_{j k}\right)$ be a sublinear MusielakOrlicz function and let $A=\left(a_{n m j k}\right)$ be a nonnegative four-dimensional $R H$-regular matrix. Suppose that $\beta=$ $\lim _{t \rightarrow \infty}\left(F_{j k}(t) / t\right)<\infty$. Then

$$
W^{2}\left(A, u, \Delta_{s}^{r}, p\right)=W^{2}\left(A, \mathscr{F}, u, \Delta_{s}^{r}, p\right) .
$$

Proof. In order to prove that $W^{2}\left(A, u, \Delta_{s}^{r}, p\right)=W^{2}(A, \mathscr{F}, u$, $\left.\Delta_{s}^{r}, p\right)$, it is sufficient to show that $W^{2}\left(A, \mathscr{F}, u, \Delta_{s}^{r}, p\right) \quad c$ $W^{2}\left(A, u, \Delta_{s}^{r}, p\right)$. Now, let $\beta>0$. By definition of $\beta$, we have $F_{j k}(t) \geq \beta t$ for all $t \geq 0$. Since $\beta>0$, we have $t \leq(1 / \beta) F_{j k}(t)$ for all $t \geq 0$. Let $x=\left(x_{j k}\right) \in W^{2}\left(A, \mathscr{F}, u, \Delta_{s}^{r}, p\right)$. Thus, we have

$$
\begin{aligned}
& \sum_{j, k=0,0}^{\infty, \infty} a_{n m j k}\left[\left(u_{j k}\left|\Delta_{s}^{r} x_{j k}-L\right|\right)^{p_{j k}}\right] \\
& \quad \leq \frac{1}{\beta} \sum_{j, k=0,0}^{\infty, \infty} a_{n m j k}\left[F_{j k}\left(u_{j k}\left|\Delta_{s}^{r} x_{j k}-L\right|\right)^{p_{j k}}\right],
\end{aligned}
$$

which implies that $x=\left(x_{j k}\right) \in W^{2}\left(A, u, \Delta_{s}^{r}, p\right)$. This completes the proof.

Theorem 13. (i) Let $0<\inf p_{j k}<p_{j k} \leq 1$. Then

$$
W^{2}\left(A, \mathscr{F}, u, \Delta_{s}^{r}, p\right) \subseteq W^{2}\left(A, \mathscr{F}, u, \Delta_{s}^{r}\right) .
$$

(ii) Let $1 \leq p_{j k} \leq \sup p_{j k}<\infty$. Then

$$
W^{2}\left(A, \mathscr{F}, u, \Delta_{s}^{r}\right) \subseteq W^{2}\left(A, \mathscr{F}, u, \Delta_{s}^{r}, p\right) .
$$

Proof. (i) Let $x=\left(x_{j k}\right) \in W^{2}\left(A, \mathscr{F}, u, \Delta_{s}^{r}, p\right)$. Then since $0<$ inf $p_{j k}<p_{j k} \leq 1$, we obtain the following:

$$
\begin{aligned}
& \sum_{j, k=0,0}^{\infty, \infty} a_{n m j k}\left[F_{j k}\left(u_{j k}\left|\Delta_{s}^{r} x_{j k}-L\right|\right)\right] \\
& \quad \leq \sum_{j, k=0,0}^{\infty, \infty} a_{n m j k}\left[F_{j k}\left(u_{j k}\left|\Delta_{s}^{r} x_{j k}-L\right|\right)^{p_{j k}}\right] .
\end{aligned}
$$

Thus $x=\left(x_{j k}\right) \in W^{2}\left(A, \mathscr{F}, u, \Delta_{s}^{r}\right)$.

(ii) Let $p_{j k} \geq 1$ for each $j$ and $k$ and $\sup p_{j k}<\infty$. Let $x=\left(x_{j k}\right) \in W^{2}\left(A, \mathscr{F}, u, \Delta_{s}^{r}\right)$. Then for each $0<\epsilon<1$ there exists a positive integer $N$ such that

$$
\sum_{j, k=0,0}^{\infty, \infty} a_{n m j k}\left[F_{j k}\left(u_{j k}\left|\Delta_{s}^{r} x_{j k}-L\right|\right)\right] \leq \epsilon<1 \quad \forall m, n \geq N .
$$


This implies that

$$
\begin{aligned}
& \sum_{j, k=0,0}^{\infty, \infty} a_{n m j k}\left[F_{j k}\left(u_{j k}\left|\Delta_{s}^{r} x_{j k}-L\right|\right)^{p_{j k}}\right] \\
& \leq \sum_{j, k=0,0}^{\infty, \infty} a_{n m j k}\left[F_{j k}\left(u_{j k}\left|\Delta_{s}^{r} x_{j k}-L\right|\right)\right] .
\end{aligned}
$$

Therefore $x=\left(x_{j k}\right) \in W^{2}\left(A, \mathscr{F}, u, \Delta_{s}^{r}, p\right)$. This completes the proof.

Lemma 14. Let $\mathscr{F}=\left(F_{j k}\right)$ be a sublinear Musielak-Orlicz function which satisfies the $\Delta_{2}$-condition and let $A=\left(a_{n m j k}\right)$ be a nonnegative four-dimensional $R H$-regular matrix. Then $W_{0}^{2}\left(A, \mathscr{F}, u, \Delta_{s}^{r}, p\right) \cap l_{\infty}^{2}$ is an ideal in $l_{\infty}^{2}$.

Proof. Let $x \in W_{0}^{2}\left(A, \mathscr{F}, u, \Delta_{s}^{r}, p\right) \cap l_{\infty}^{2}$ and $y \in l_{\infty}^{2}$. We need to show that $x y \in W_{0}^{2}\left(A, \mathscr{F}, u, \Delta_{s}^{r}, p\right) \cap l_{\infty}^{2}$. Since $y \in l_{\infty}^{2}$, there exists $T_{1}>1$ such that $\|y\|<T_{1}$. In this case $\left|x_{j k} y_{j k}\right|<$ $T_{1}\left|x_{j k}\right|$ for all $j, k$. Since $\mathscr{F}$ is nondecreasing and satisfies $\Delta_{2}-$ condition, we have

$$
\begin{aligned}
{\left[F_{j k}\left(u_{j k}\left|\Delta_{s}^{r}\left(x_{j k} y_{j k}\right)\right|\right)^{p_{j k}}\right] } & <\left[F_{j k}\left(u_{j k} T_{1}\left|\Delta_{s}^{r} x_{j k}\right|\right)^{p_{j k}}\right] \\
& \leq T\left(T_{1}\right)\left[F_{j k}\left(u_{j k}\left|\Delta_{s}^{r} x_{j k}\right|\right)^{p_{j k}}\right],
\end{aligned}
$$

for all $j, k$ and $T>0$. Therefore $\lim _{n, m} \sum_{j, k} a_{n m j k}\left[F_{j k}\right.$ $\left.\left(u_{j k}\left|\Delta_{s}^{r}\left(x_{j k} y_{j k}\right)\right|\right)^{p_{j k}}\right]=0$. Thus $x y \in W_{0}^{2}\left(A, \mathscr{F}, u, \Delta_{s}^{r}, p\right) \cap l_{\infty}^{2}$. This completes the proof.

Lemma 15. Let $G$ be an ideal in $l_{\infty}^{2}$ and let $x=\left(x_{j k}\right) \in l_{\infty}^{2}$. Then $x$ is in the closure of $G$ in $l_{\infty}^{2}$ if and only if $\chi_{S(x ; \epsilon)} \in G$ for all $\epsilon>0$.

Proof. Let $x$ be in the closure of $G$ and let $\epsilon>0$ be given. Suppose that $z=\left(z_{j k}\right) \in G$ such that $\|x-z\|<\epsilon / 2$ and observe that $S(x ; \epsilon) \subseteq S(z ; \epsilon / 2)$. Define a double sequence $y=$ $\left(y_{j k}\right) \in l_{\infty}^{2}$ by

$$
y_{j k}= \begin{cases}\frac{1}{z_{j k}}, & \text { if }\left|z_{j k}\right| \geqq \frac{\epsilon}{2}, \\ 0, & \text { otherwise. }\end{cases}
$$

Clearly $y z=\chi_{S(z ; \epsilon / 2)} \in G$. Since $S(x ; \epsilon) \subseteq S(z ; \epsilon / 2)$ and $\chi_{S(x ; \epsilon)} \in l_{\infty}^{2}$, hence $\chi_{S(x ; \epsilon)} \chi_{S(z ; \epsilon / 2)}=\chi_{S(x ; \epsilon)} \in G$.

Conversely, if $x \in l_{\infty}^{2}$ then $\left\|x-x \chi_{S(x ; \epsilon)}\right\|<\epsilon$. It follows that $\chi_{S(x ; \epsilon)} \in G$ for all $\epsilon>0$; then $x$ is in the closure of $G$.

Lemma 16. If $A$ is a nonnegative four-dimensional $R H$ regular matrix, then $W_{0}^{2}\left(A, u, \Delta_{s}^{r}, p\right) \cap l_{\infty}^{2}$ is a closed ideal in $l_{\infty}^{2}$
Proof. We have $W_{0}^{2}\left(A, \mathscr{F}, u, \Delta_{s}^{r}, p\right) \cap l_{\infty}^{2} \subset l_{\infty}^{2}$ and it is clear that $W_{0}^{2}\left(A, \mathscr{F}, u, \Delta_{s}^{r}, p\right) \cap l_{\infty}^{2} \neq 0$. For $x, y \in W_{0}^{2}(A, \mathscr{F}, u$, $\left.\Delta_{s}^{r}, p\right) \cap l_{\infty}^{2}$, we get $\left|x_{j k}+y_{j k}\right|<\left|x_{j k}\right|+\left|y_{j k}\right|$. Now, we have

$$
\begin{aligned}
& {\left[F_{j k}\left(u_{j k}\left|\Delta_{s}^{r}\left(x_{j k}+y_{j k}\right)\right|\right)^{p_{j k}}\right]} \\
& \quad \leq\left[F_{j k}\left(u_{j k}\left|\Delta_{s}^{r} x_{j k}\right|+\left|\Delta_{s}^{r} y_{j k}\right|\right)^{p_{j k}}\right] \\
& \quad<\frac{1}{2}\left[F_{j k}\left(u_{j k} 2\left|\Delta_{s}^{r} x_{j k}\right|\right)^{p_{j k}}\right]+\frac{1}{2}\left[F_{j k}\left(u_{j k} 2\left|\Delta_{s}^{r} y_{j k}\right|\right)^{p_{j k}}\right] \\
& \quad<\frac{1}{2} K_{1}\left[F_{j k}\left(u_{j k}\left|\Delta_{s}^{r} x_{j k}\right|\right)^{p_{j k}}\right]+\frac{1}{2} K_{2}\left[F_{j k}\left(u_{j k}\left|\Delta_{s}^{r} y_{j k}\right|\right)^{p_{j k}}\right]
\end{aligned}
$$

by the $\Delta_{2}$-condition and the convexity of $F$. Since

$$
\begin{aligned}
\sum_{j, k} a_{n m j k}\left[F_{j k}\left(u_{j k}\left|\Delta_{s}^{r}\left(x_{j k}+y_{j k}\right)\right|\right)^{p_{j k}}\right] \\
\leq \frac{1}{2} K \sum_{j, k} a_{n m j k}\left[F_{j k}\left(u_{j k}\left|\Delta_{s}^{r} x_{j k}\right|\right)^{p_{j k}}\right] \\
+\frac{1}{2} K \sum_{j, k} a_{n m j k}\left[F_{j k}\left(u_{j k}\left|\Delta_{s}^{r} y_{j k}\right|\right)^{p_{j k}}\right],
\end{aligned}
$$

where $K=\max \left\{K_{1}, K_{2}\right\}$, so $x+y, x-y \in W_{0}^{2}\left(A, \mathscr{F}, u, \Delta_{s}^{r}, p\right) \cap$ $l_{\infty}^{2}$

Let $x \in W_{0}^{2}\left(A, \mathscr{F}, u, \Delta_{s}^{r}, p\right) \cap l_{\infty}^{2}$ and $y \in l_{\infty}^{2}$. Thus, there exists a positive integer $K$, so that, for every $j, k$, we have $\left|x_{j k} y_{j k}\right| \leq K\left|x_{j k}\right|$. Therefore

$$
\begin{aligned}
{\left[F_{j k}\left(u_{j k}\left|\Delta_{s}^{r}\left(x_{j k} y_{j k}\right)\right|\right)^{p_{j k}}\right] } & \leq\left[F_{j k}\left(u_{j k} K\left|\Delta_{s}^{r} x_{j k}\right|\right)^{p_{j k}}\right] \\
& \leq T\left[F_{j k}\left(u_{j k}\left|\Delta_{s}^{r} x_{j k}\right|\right)^{p_{j k}}\right],
\end{aligned}
$$

and so

$$
\begin{gathered}
\sum_{j, k} a_{n m j k}\left[F_{j k}\left(u_{j k}\left|\Delta_{s}^{r}\left(x_{j k} y_{j k}\right)\right|\right)^{p_{j k}}\right] \\
\leq T \sum_{j, k} a_{n m j k}\left[F_{j k}\left(u_{j k}\left|\Delta_{s}^{r} x_{j k}\right|\right)^{p_{j k}}\right] .
\end{gathered}
$$

Hence $x y \in W_{0}^{2}\left(A, \mathscr{F}, u, \Delta_{s}^{r}, p\right) \cap l_{\infty}^{2}$. So $W_{0}^{2}\left(A, \mathscr{F}, u, \Delta_{s}^{r}, p\right) \cap$ $l_{\infty}^{2}$ is an ideal in $l_{\infty}^{2}$ for a Musielak-Orlicz function which satisfies the $\Delta_{2}$-condition.

Now, we have to show that $W_{0}^{2}\left(A, \mathscr{F}, u, \Delta_{s}^{r}, p\right) \cap l_{\infty}^{2}$ is closed. Let $x \in \overline{W_{0}^{2}\left(A, \mathscr{F}, u, \Delta_{s}^{r}, p\right) \cap l_{\infty}^{2}}$; there exists $x^{c d}=$ $x_{j k}^{c d} \in W_{0}^{2}\left(A, \mathscr{F}, u, \Delta_{s}^{r}, p\right) \cap l_{\infty}^{2}$ such that $x^{c d} \rightarrow x \in l_{\infty}^{2}$. 
For every $\epsilon>0$ there exists $N_{1}(\epsilon) \in \mathbb{N}$ such that, for all $c, d>N_{1}(\epsilon),\left|\Delta_{s}^{r} x^{c d}-\Delta_{s}^{r} x\right|<\epsilon$. Now, for $\epsilon>0$, we have

$$
\begin{aligned}
\sum_{j, k} a_{n m j k}\left[F_{j k}\left(u_{j k}\left|\Delta_{s}^{r} x_{j k}\right|\right)^{p_{j k}}\right] & \\
= & \sum_{j, k} a_{n m j k}\left[F_{j k}\left(u_{j k}\left|\Delta_{s}^{r} x_{j k}-\Delta_{s}^{r} x_{j k}^{c d}+\Delta_{s}^{r} x_{j k}^{c d}\right|\right)^{p_{j k}}\right] \\
\leq & \sum_{j, k} a_{n m j k}\left[F_{j k}\left(u_{j k}\left|\Delta_{s}^{r} x_{j k}-\Delta_{s}^{r} x_{j k}^{c d}\right|+\left|\Delta_{s}^{r} x_{j k}^{c d}\right|\right) p^{p_{j k}}\right] \\
\leq & \frac{1}{2} \sum_{j, k} a_{n m j k}\left[F_{j k}\left(u_{j k} 2\left|\Delta_{s}^{r} x_{j k}-\Delta_{s}^{r} x_{j k}^{c d}\right|\right)^{p_{j k}}\right] \\
& +\frac{1}{2} \sum_{j, k} a_{n m j k}\left[F_{j k}\left(u_{j k} 2\left|\Delta_{s}^{r} x_{j k}^{c d}\right|\right)^{p_{j k}}\right] \\
\leq & \frac{1}{2} K F_{j k}(\epsilon) \sum_{j, k} a_{n m j k}+\frac{1}{2} K \sum_{j, k} a_{n m j k}\left[F_{j k}\left(u_{j k}\left|\Delta_{s}^{r} x_{j k}^{c d}\right|\right)^{p_{j k}}\right] .
\end{aligned}
$$

Since $x^{c d} \in W_{0}^{2}\left(A, \mathscr{F}, u, \Delta_{s}^{r}, p\right) \cap l_{\infty}^{2}$ and $A$ is $R H$-regular, we get

$$
\lim _{n, m} \sum_{j, k} a_{n m j k}\left[F_{j k}\left(u_{j k}\left|\Delta_{s}^{r} x_{j k}\right|\right)^{p_{j k}}\right]=0
$$

so $x \in W_{0}^{2}\left(A, \mathscr{F}, u, \Delta_{s}^{r}, p\right) \cap l_{\infty}^{2}$. This completes the proof.

Theorem 17. Let $x=\left(x_{j k}\right)$ be a bounded sequence, $\mathscr{F}=\left(F_{j k}\right)$ a sublinear Musielak-Orlicz function which satisfies the $\Delta_{2}-$ condition, and $A$ a nonnegative four-dimensional $R H$-regular matrix. Then $W^{2}\left(A, \mathscr{F}, u, \Delta_{s}^{r}, p\right) \cap l_{\infty}^{2}=W^{2}\left(A, u, \Delta_{s}^{r}, p\right) \cap l_{\infty}^{2}$.

Proof. Without loss of generality we may take $L=0$ and establish

$$
W_{0}^{2}\left(A, \mathscr{F}, u, \Delta_{s}^{r}, p\right) \cap l_{\infty}^{2}=W_{0}^{2}\left(A, u, \Delta_{s}^{r}, p\right) \cap l_{\infty}^{2}
$$

Since $W_{0}^{2}\left(A, u, \Delta_{s}^{r}, p\right) \subseteq W_{0}^{2}\left(A, \mathscr{F}, u, \Delta_{s}^{r}, p\right)$, therefore $W_{0}^{2}(A$, $\left.u, \Delta_{s}^{r}, p\right) \cap l_{\infty}^{2} \subseteq W_{0}^{2}\left(A, \mathscr{F}, u, \Delta_{s}^{r}, p\right) \cap l_{\infty}^{2}$. We need to show that $W_{0}^{2}\left(A, \mathscr{F}, u, \Delta_{s}^{r}, p\right) \cap l_{\infty}^{2} \subseteq W_{0}^{2}\left(A, u, \Delta_{s}^{r}, p\right) \cap l_{\infty}^{2}$. Notice that if $S \subset \mathbb{N} \times \mathbb{N}$, then

$$
\sum_{j, k} a_{n m j k}\left[F_{j k}\left(\chi_{S}(j, k)\right)^{p_{j k}}\right]=F_{j k}(1) \sum_{j, k} a_{n m j k}\left(\chi_{S}(j, k)\right)^{p_{j k}}
$$

for all $n, m$. Observe that $\chi_{S}(j, k) \in W_{0}^{2}\left(A, u, \Delta_{s}^{r}, p\right) \cap l_{\infty}^{2}$ whenever $x \in W_{0}^{2}\left(A, \mathscr{F}, u, \Delta_{s}^{r}, p\right) \cap l_{\infty}^{2}$ by Lemmas 14 and 15 , so

$$
W_{0}^{2}\left(A, \mathscr{F}, u, \Delta_{s}^{r}, p\right) \cap l_{\infty}^{2} \subseteq W_{0}^{2}\left(A, u, \Delta_{s}^{r}, p\right) \cap l_{\infty}^{2}
$$

The proof is complete.

\section{Conflict of Interests}

The authors declare that there is no conflict of interests regarding the publication of this paper.

\section{Acknowledgment}

The authors gratefully acknowledge the financial support from King Abdulaziz University, Jeddah, Saudi Arabia.

\section{References}

[1] G. H. Hardy, "On the convergence of certain multiple series," Proceedings of the Cambridge Philosophical Society, vol. 19, pp. 86-95, 1917.

[2] T. J. Bromwich, An Introduction to the Theory of Infinite Series, Macmillan and co. Ltd, New York, NY, USA, 1965.

[3] F. Móricz, "Extensions of the spaces $c$ and $c_{0}$ from single to double sequences," Acta Mathematica Hungarica, vol. 57, no. 1-2, pp. 129-136, 1991.

[4] F. Móricz and B. E. Rhoades, "Almost convergence of double sequences and strong regularity of summability matrices," Mathematical Proceedings of the Cambridge Philosophical Society, vol. 104, no. 2, pp. 283-294, 1988.

[5] M. Başarır and O. Sonalcan, "On some double sequence spaces," Indian Academy of Mathematics, vol. 21, no. 2, pp. 193-200, 1999.

[6] M. Mursaleen and S. A. Mohiuddine, "Regularly $\sigma$-conservative and $\sigma$-coercive four dimensional matrices," Computers \& Mathematics with Applications, vol. 56, no. 6, pp. 1580-1586, 2008.

[7] M. Mursaleen and S. A. Mohiuddine, "On $\sigma$-conservative and boundedly $\sigma$-conservative four-dimensional matrices," Computers \& Mathematics with Applications, vol. 59, no. 2, pp. 880885, 2010.

[8] Mursaleen and S. A. Mohiuddine, "Double $\sigma$-multiplicative matrices," Journal of Mathematical Analysis and Applications, vol. 327, no. 2, pp. 991-996, 2007.

[9] M. Mursaleen, "Almost strongly regular matrices and a core theorem for double sequences," Journal of Mathematical Analysis and Applications, vol. 293, no. 2, pp. 523-531, 2004.

[10] M. Mursaleen and O. H. H. Edely, "Almost convergence and a core theorem for double sequences," Journal of Mathematical Analysis and Applications, vol. 293, no. 2, pp. 532-540, 2004.

[11] S. A. Mohiuddine and A. Alotaibi, "Almost conservative fourdimensional matrices through de la Vallée-Poussin mean," Abstract and Applied Analysis, vol. 2014, Article ID 412974, 6 pages, 2014.

[12] B. Altay and F. Başar, "Some new spaces of double sequences," Journal of Mathematical Analysis and Applications, vol. 309, no. 1, pp. 70-90, 2005.

[13] F. Başar and Y. Sever, "The space $L_{q}$ of double sequences," Mathematical Journal of Okayama University, vol. 51, pp. 149$157,2009$.

[14] M. Mursaleen and S. A. Mohiuddine, "Some matrix transformations of convex and paranormed sequence spaces into the spaces of invariant means," Journal of Function Spaces and Applications, vol. 2012, Article ID 612671, 10 pages, 2012.

[15] S. A. Mohiuddine and A. Alotaibi, "Some spaces of double sequences obtained through invariant mean and related concepts," Abstract and Applied Analysis, vol. 2013, Article ID 507950, 11 pages, 2013. 
[16] K. Demirci, "Strong $A$-summability and $A$-statistical convergence," Indian Journal of Pure and Applied Mathematics, vol. 27, no. 6, pp. 589-593, 1996.

[17] M. Mursaleen and S. A. Mohiuddine, "Some new double sequence spaces of invariant means," Glasnik Matematički, vol. 45, no. 1, pp. 139-153, 2010.

[18] S. D. Parashar and B. Choudhary, "Sequence spaces defined by Orlicz functions," Indian Journal of Pure and Applied Mathematics, vol. 25, no. 4, pp. 419-428, 1994.

[19] H. Kizmaz, "On certain sequence spaces," Canadian Mathematical Bulletin, vol. 24, no. 2, pp. 169-176, 1981.

[20] M. Et and R. Çolak, "On some generalized difference sequence spaces," Soochow Journal of Mathematics, vol. 21, no. 4, pp. 377386, 1995.

[21] M. Et, "Spaces of Cesàro difference sequences of order $r$ defined by a modulus function in a locally convex space," Taiwanese Journal of Mathematics, vol. 10, no. 4, pp. 865-879, 2006.

[22] M. Et, "Generalized Cesàro difference sequence spaces of nonabsolute type involving lacunary sequences," Applied Mathematics and Computation, vol. 219, no. 17, pp. 9372-9376, 2013.

[23] K. Raj, A. K. Sharma, and S. K. Sharma, "A sequence space defined by Musielak-Orlicz function," International Journal of Pure and Applied Mathematics, vol. 67, no. 4, pp. 475-484, 2011.

[24] K. Raj, S. Jamwal, and S. K. Sharma, "New classes of generalized sequence spaces defined by an Orlicz function," Journal of Computational Analysis and Applications, vol. 15, no. 4, pp. 730737, 2013.

[25] K. Raj and S. K. Sharma, "Some generalized difference double sequence spaces defined by a sequence of Orlicz-functions," Cubo, vol. 14, no. 3, pp. 167-190, 2012.

[26] B. C. Tripathy, "Generalized difference paranormed statistically convergent sequences defined by Orlicz function in a locally convex space," Soochow Journal of Mathematics, vol. 30, no. 4, pp. 431-446, 2004.

[27] M. Et, Y. Altin, B. Choudhary, and B. C. Tripathy, "On some classes of sequences defined by sequences of Orlicz functions," Mathematical Inequalities \& Applications, vol. 9, no. 2, pp. 335342, 2006.

[28] J. Lindenstrauss and L. Tzafriri, "On Orlicz sequence spaces," Israel Journal of Mathematics, vol. 10, pp. 379-390, 1971.

[29] L. Maligranda, Orlicz Spaces and Interpolation, vol. 5 of Seminars in Mathematics, Polish Academy of Science, 1989.

[30] J. Musielak, Orlicz Spaces and Modular Spaces, vol. 1034 of Lecture Notes in Mathematics, Springer, Berlin, Germany, 1983.

[31] A. Pringsheim, "Zur Theorie der zweifach unendlichen Zahlenfolgen," Mathematische Annalen, vol. 53, no. 3, pp. 289-321, 1900.

[32] R. G. Cooke, Infinite Matrices and Sequence Spaces, Macmillan \& Co., London, UK, 1950.

[33] G. M. Robison, "Divergent double sequences and series," Transactions of the American Mathematical Society, vol. 28, no. 1, pp. 50-73, 1926.

[34] H. J. Hamilton, “Transformations of multiple sequences," Duke Mathematical Journal, vol. 2, no. 1, pp. 29-60, 1936.

[35] I. J. Maddox, Elements of Functional Analysis, Cambridge University Press, Cambridge, UK, 2nd edition, 1988.

[36] S. Simons, "The sequence spaces $l\left(p_{v}\right)$ and $m\left(p_{v}\right)$," Proceedings of the London Mathematical Society, vol. 15, pp. 422-436, 1965.

[37] T. Yurdakadim and E. Tas, "Double sequences and Orlicz functions," Periodica Mathematica Hungarica, vol. 67, no. 1, pp. 47-54, 2013. 


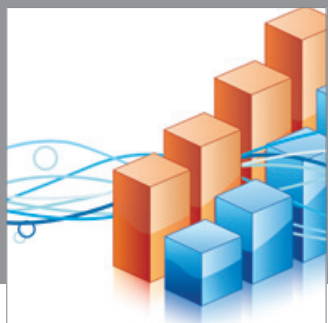

Advances in

Operations Research

mansans

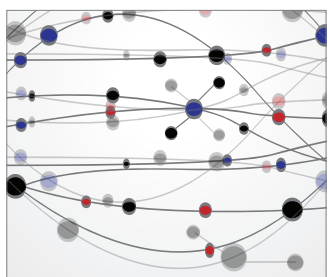

The Scientific World Journal
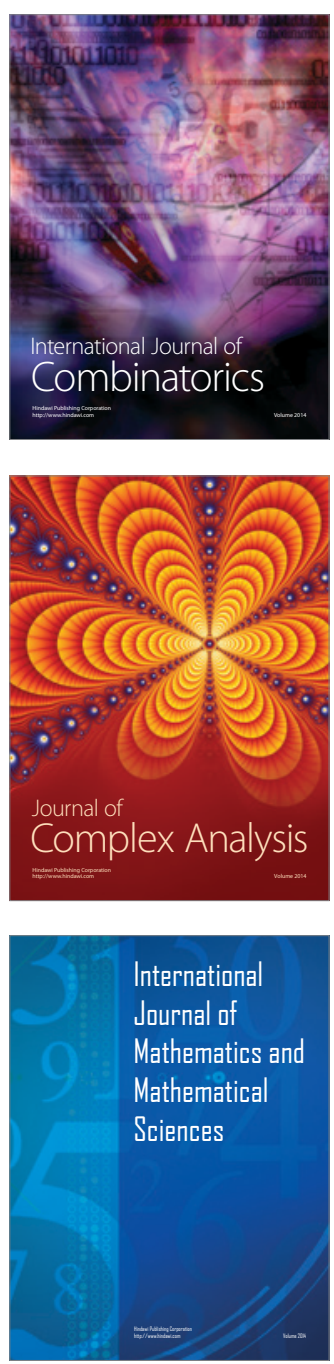
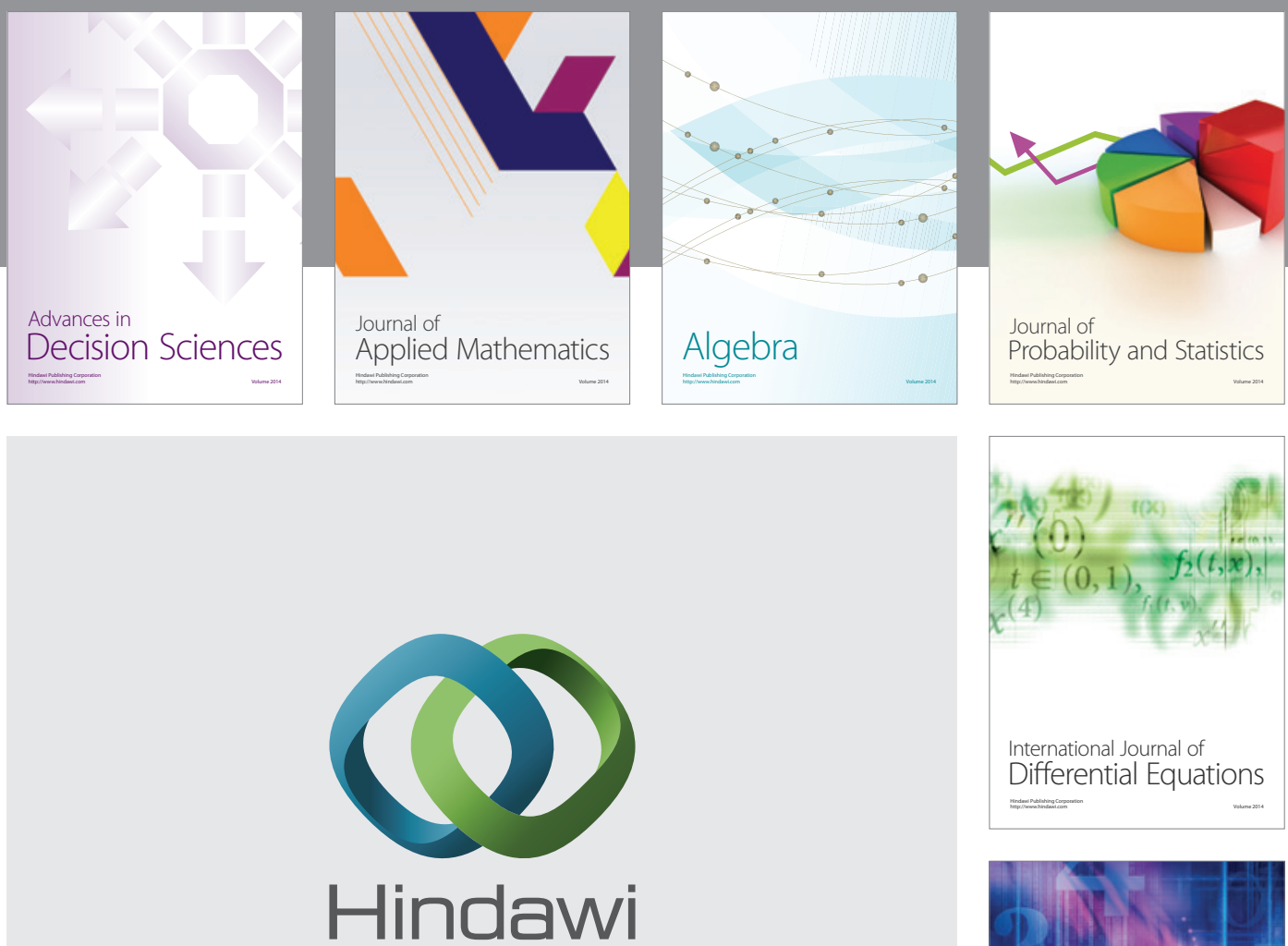

Submit your manuscripts at http://www.hindawi.com
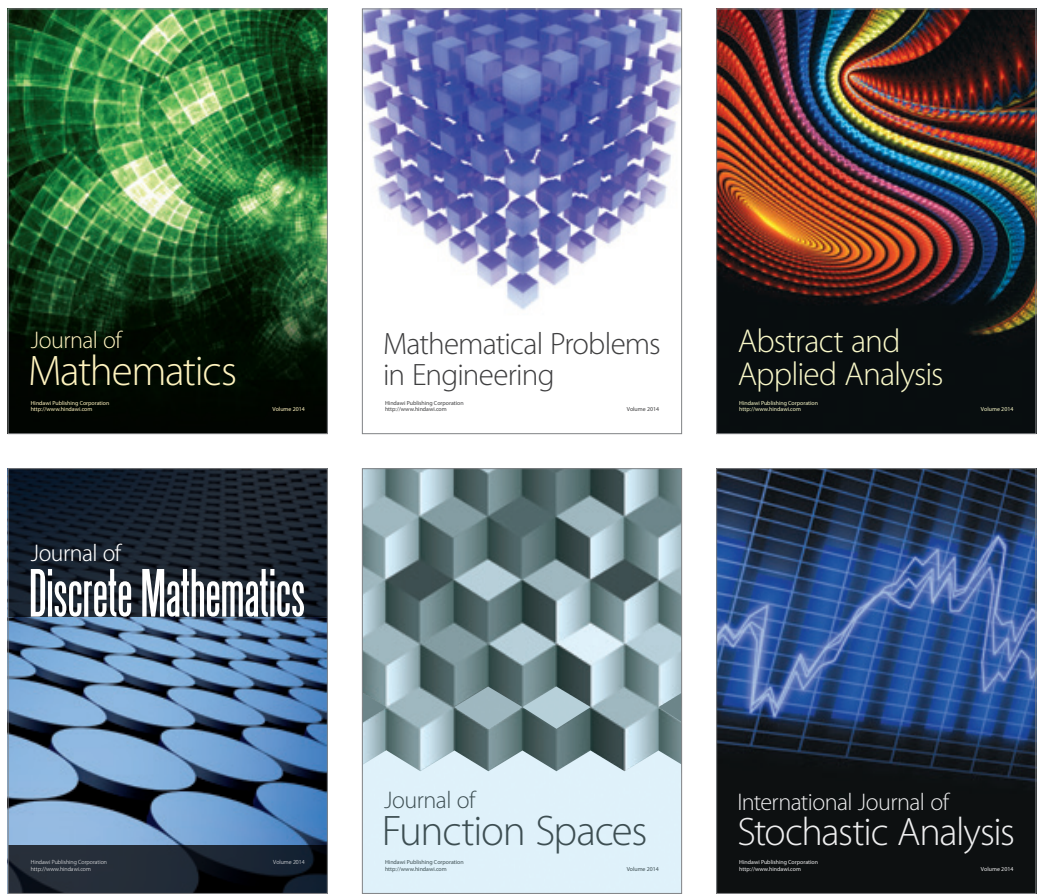

Journal of

Function Spaces

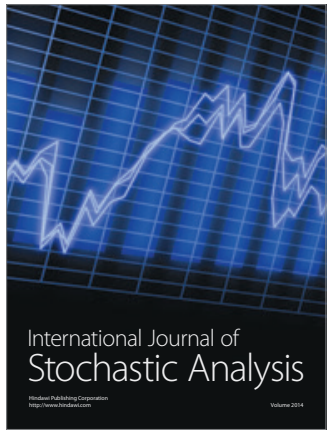

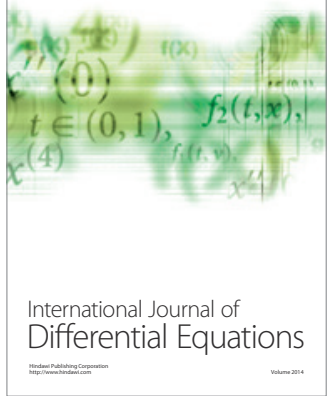
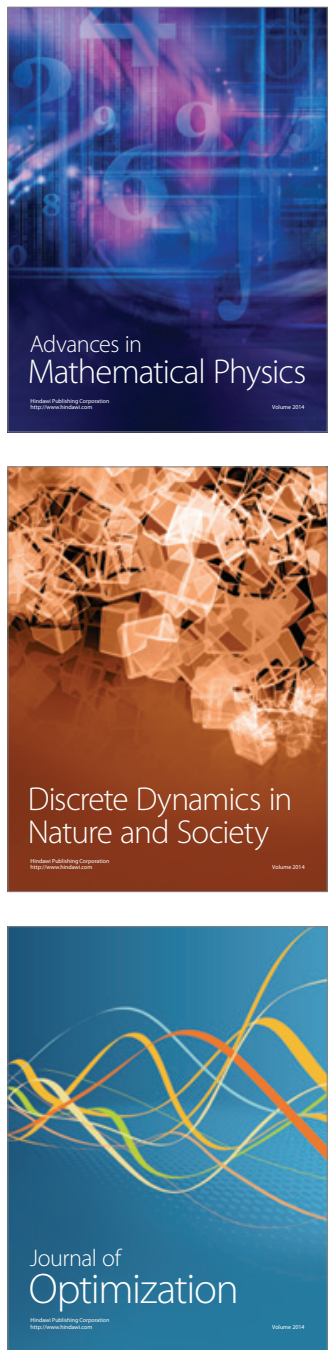